\title{
Intersection Properties of Invariant Manifolds in Certain Twist Maps
}

\author{
J. J. P. Veerman ${ }^{1}$ and F. M. Tangerman ${ }^{2}$ \\ 1 Institute for the Mathematical Sciences, SUNY at Stony Brook, Stony Brook, NY 11794, USA \\ 2 Mathematics Department, SUNY at Stony Brook, Stony Brook, NY 11794, USA
}

Received June 7, 1990

\begin{abstract}
We consider the space $N$ of $C^{2}$ twist maps that satisfy the following requirements. The action is the sum of a purely quadratic term and a periodic potential times a constant $k$ (hereafter called the nonlinearity). The potential restricted to the unit circle is bimodal, i.e. has one local minimum and one local maximum. The following statements are proven for maps in $N$ with nonlinearity $k$ large enough. The intersection of the unstable and stable invariant manifolds to the hyperbolic minimizing periodic points contains minimizing homoclinic points. Consider two finite pieces of these manifolds that connect two adjacent homoclinic minimizing points (hereafter called fundamental domains). We prove that all such fundamental domains have precisely one point in their intersection (the Single Intersection theorem).

In addition, we show that limit points of minimizing points are recurrent, which implies that Aubry Mather sets (with irrational rotation number) are contained in diamonds formed by local stable and unstable manifolds of nearby minimizing periodic orbits (the Diamond Configuration theorem). Another corollary concerns the intersection of the minimax orbits with certain symmetry lines of the map.
\end{abstract}

\section{Introduction and Results}

The main objective of this work is to bound the number of ways that stable and unstable manifolds of minimizing orbits can intersect each other. We do this for a class of maps whose members are close (in the $C^{2}$-topology) to a standard map with large $k$.

We will consider maps generated by the action:

$$
h\left(x, x^{\prime}\right)=1 / 2\left(x-x^{\prime}\right)^{2}+k V(x),
$$

where

$$
V \in C^{3}\left(S^{1}\right)
$$


$V^{\prime}$ has a unique minimum $x_{\min }$ and unique maximum $x_{\max }$,

$$
V^{\prime \prime}\left(x_{\min }\right)=1 \text { and } V^{\prime \prime}\left(x_{\max }\right)=-1 \text {. }
$$

The first of these last two requirements can be achieved by normalizing the constant $k$. The second requirement is to avoid inessential constants in the exposition; the theory can be written up taking this constant into account. We remark that the theory developed here can also be applied to multimodal potentials, albeit with different conclusions. The generalization of the theory to actions that do not have the form of a quadratic piece plus a "potential" is more problematic since we have relied upon the "Laplacian" form of the map [e.g. Eq. (2.1)]. However, it appears that serious problems only arise if $\partial_{x} \partial_{x} h\left(x, x^{\prime}\right)$ is not bounded away from zero. In this article we have restricted ourselves to the simplest case given by Eq. (1.1a).

We have:

$$
f_{k}:\left\{\begin{array}{l}
x \rightarrow x+y+k V^{\prime}(x) \\
y \rightarrow y+k V^{\prime}(x)
\end{array},\right.
$$

and here $f$ maps $R^{2}$ to itself. We adopt the convention that Aubry Mather sets, henceforth denoted by $E_{\rho}$, correspond to the minima with irrational rotation number of the functional

$$
H=\sum_{i=-\infty}^{+\infty} h\left(x_{i}, x_{i+1}\right)
$$

(see Aubry 1983; Mather 1986). The other minima of $H$ are denoted by $E_{p / q}$ for the periodic orbits, $E_{p / q}$ for the advancing orbits, and $E_{p / q}$ for the receding orbits. The map $f$ can regarded as the lift to the universal covering of either a map of the torus to itself or of the cylinder to itself.

We will now define the notion of separating curves (see Veerman and Tangerman 1990), which will play a major role in the exposition. For each $p / q+$ or $p / q-$, separating curves $\gamma(p / q+)$ or $\gamma(p / q-)$ are defined as follows. Let $s_{1}$ be a point on $E_{p / q+}$ or $E_{p / q-}$. Connect $s_{1}$ to the neighboring points $p_{1}$ and $p_{2}$ of $E_{p / q}$ along their invariant manifolds (see Fig. 1.1). Repeat this for all neighboring pairs of points of $E_{p / q}$. Note, that these curves and components depend on the choice of $q$ points $s_{1}, \ldots, s_{q}$ in $E_{p / q+}$, respectively, $E_{p / q-}$. In Sect. 2, it will be shown that we can choose these points so that the separating curves are actually graphs over $x$ separating $R^{2}$ in an upper component containing $+\infty$ and a lower component containing $-\infty$. We will also show (Sect. 4 ) that the Cantor sets $E_{\varrho}$ are contained in the union of open, diamond-shaped regions whose boundary is formed by the local invariant manifolds that are part of these curves (Diamond Configuration).

The following conventions will be adhered to throughout the article. Consider the projections of the Aubry Mather sets on the $x$-axis. By a "gap" $G$ in $E_{\varrho}$, $\varrho \in\{R, Q-, Q+\}$, we mean (see Katok 1982b) a pair of points in $E_{\varrho}$, whose projections bound an interval that contains no point of the projection of $E_{\varrho}$. The meaning of $f^{i}(G)$ is then also clear. Denote the finite pieces of invariant manifolds to $E_{\varrho}$ that connect the endpoints of a gap $G$ in $E_{\varrho}$ by $W^{s}(G)$ and $W^{u}(G)$. We will say that $f$ satisfies the Single Intersection hypothesis if all $E_{p / q}, E_{p / q-}$, and $E_{p / q+}$ are unique, and if, for a gap $G$ in $E_{p / q+}$ or $E_{p / q-}, W^{u}(G)$ intersected with $W^{s}(G)$ contains a single point (which then has to be the minimax) besides the endpoints, see Fig. 1.1. 


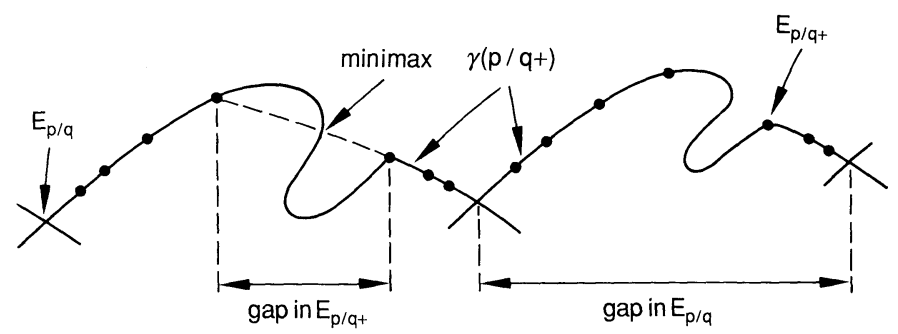

Fig. 1.1. Separating curves and gaps

There are two motivations for the present work. The first one is very prosaic. In an earlier work (Veerman and Tangerman 1990), we posed the Single Intersection hypothesis in order to prove a number of results concerning periodic and quasiperiodic behavior in 2-dimensional area preserving twist maps. Given the complex character of the hypothesis and the fact that all the support we could conjure up for it was numerical, it seemed worthwhile to prove that this hypothesis holds for at least a limited class of maps.

In that paper, we also needed to assume that limit-points with irrational rotation number of minimizing orbits are recurrent, but that conjecture had been stated before by Bangert (1986). These results will spin off from the reasoning we set up to prove the Single Intersection hypothesis.

The fact that all minimax orbits have a point in common with a symmetry is very important in current numerical work (Kook and Meiss 1988), and since it follows from our reasoning, we have included it in this work.

The second, and perhaps more profound, motivation is that it appeared interesting to study other structures than just orbits and their closures, the most common objects for study. For example, details of how stable and unstable manifolds intersect each other may at first seem an unsurpassably messy problem. However, as results from the considerations in this work, not all aspects of that problem are as nasty as one might imagine.

Section 2 contains as main result the statement that for large $k$, we can draw separating curves $\gamma(p / q+)$ which are uniformly Lipschitz and similarly for the family of curves $\gamma(p / q-)$. In Sect. 3, we prove existence and uniqueness of certain periodic orbits, and in Sect. 4 we do the same for certain homoclinic orbits. The results of Sect. 4 also include the main statement of this work. In Sect. 5, it is proved that minimaxes have a point in common with a symmetry line. It also contains some results concerning the geometry of Aubry Mather sets (the Diamond Configuration is proved). In Sect. 6, we obtain an estimate, in the case of the standard map, for the lower bound of the nonlinearity $k$ for which our main conclusions hold. Finally, in the appendices we collect the linear algebra that we need to state our results. Some of the facts collected there are well-known and we make no claim to originality here: the collection is for the convenience of the reader since not all the results are self-evident or standard knowledge. Some results in the appendices admit generalizations (for example see MacKay and Meiss 1983). However, we have opted to give the statements their simplest form still suitable for our purposes. 
For future reference, we list the following:

$$
\begin{gathered}
f_{k}^{-1}:\left\{\begin{array}{l}
x \rightarrow x-y \\
y \rightarrow y-k V^{\prime}(x-y)
\end{array}\right. \\
D f(x, y)=\left(\begin{array}{cc}
1+k V^{\prime \prime}(x) & 1 \\
k V^{\prime \prime}(x) & 1
\end{array}\right), \\
D f^{-1}(x, y)=\left(\begin{array}{cc}
1 & -1 \\
-k V^{\prime \prime}(x-y) & 1+k V^{\prime \prime}(x-y)
\end{array}\right) .
\end{gathered}
$$

Finally, $f^{-1}$ is generated by action (1.1a) with $x$ and $x^{\prime}$ interchanged.

We will denote the norm $\max _{i}\left\{\left|x_{i}\right|\right\}$ in $R^{n}$ by $\mid I_{m}$ and the Euclidean norm by || . The operator norm $\sup _{x} \frac{|A x|_{m}^{i}}{|x|_{m}}$ for an operator $A$ is denoted by $\|A\|_{m}$.

\section{Orbits of Bounded Type}

In this section, we define the notion of bounded type and prove that the class of orbits we are interested in (the ones that live in the intersection of certain fundamental domains of invariant stable and unstable manifolds) are of bounded type.

Definition 2.1. Let $\left\{\left(x_{i}, y_{i}\right)\right\}_{i=-\infty}^{+\infty}$ be an orbit of $f$. The "type" $t$ of this orbit is defined as follows:

$$
t=\sup _{i \in Z}\left|x_{i}-x_{i-1}+x_{i}-x_{i+1}\right| \text {. }
$$

Remarks. According to the definition of $f$, we also have

$$
t=\sup _{i \in Z}\left|y_{i}-y_{i+1}\right| \text {. }
$$

The type of an orbit corresponds therefore with the supremum of the $2^{\text {nd }}$ differences of the $x_{i}$ and the supremum of the first differences of the $y_{i}$. Note further that if the sequence $\left\{\left|y_{i}\right|\right\}_{-\infty}^{+\infty}$ is bounded then so is the type of the orbit. For fixed $k$ all orbits under the map $f$ have bounded type. Intuitively, one expects that as $k$ increases so does the type of the orbits that can occur.

Lemma 2.2. All well-ordered orbits have type no greater than one.

Proof. If the rotation number $\varrho$ of the orbit satisfies $-1 / 2 \leqq \varrho \leqq 1 / 2$, then being wellordered implies

$$
0 \leqq x_{i}-x_{i-1} \leqq 1,
$$

from which the statement follows. For other rotation numbers, the above inequality also holds by translational invariance.

Define the projection $\pi: R^{2} \rightarrow R$ as follows:

$$
\pi(x, y)=x
$$

and denote the collection of orbits of $f_{k}$ with type $0 \leqq t \leqq T$ by $O_{T}$. 


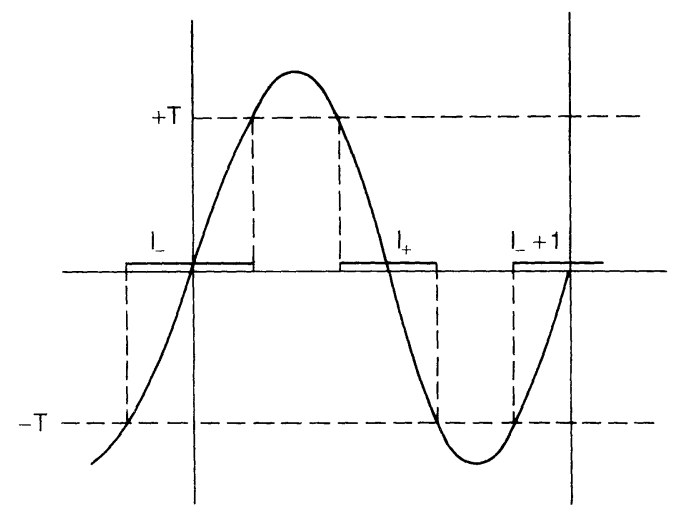

Fig. 2.1. The intervals $I_{-}$and $I_{+}$

Lemma 2.3. i) For all $T$, there is a $K_{1}(T)>0$ such that for all $k>K_{1}(T), \pi\left(O_{T}\right)$ is contained in two disjoint open intervals in plus their translates. $I_{-}(k, T)$ containing the minimum of $V$, and $I_{+}(k, T)$ containing the maximum of $V$.

ii) (Aubry 1983) For $k>K_{1}(1)$, the projection of well-ordered minimizing orbits are contained in a single interval $I_{-}(k, 1)=\left\{x|| V^{\prime}(x) \mid<1 / k\right.$ and $\left.V^{\prime \prime}(x)>-2 / k\right\}$ plus its translates.

Proof. To prove the first part, note that according to (1.1), we have that orbits are critical points of $H$. Therefore,

$$
\left(x_{i}-x_{i-1}\right)+\left(x_{i}-x_{i+1}\right)+k V^{\prime}\left(x_{i}\right)=0 \text {. }
$$

So,

$$
\left|V^{\prime}\left(x_{i}\right)\right|<T / k,
$$

see Fig. 2.1. The second part follows by observing that for minima one has

$$
\frac{\partial^{2} H}{\partial x^{2}}=2+k V^{\prime \prime}(x)>0
$$

Note that the intervals decrease monotonically in size with $k$ and $T$.

From this lemma, one concludes that all gaps of $E_{p / q+}$ and $E_{p / q_{-}}$, except for one, also project to $I_{-}$. Call this exceptional gap $G_{0}$. Define the curves:

$$
\begin{aligned}
& \gamma(p / q+)=\bigcup_{i \leqq 0} W_{+}^{u}\left(G_{i}\right) \bigcup_{i>0} W_{+}^{s}\left(G_{i}\right), \\
& \gamma(p / q-)=\bigcup_{i \leqq 0} W_{-}^{u}\left(G_{i}\right) \bigcup_{i>0} W_{-}^{s}\left(G_{i}\right),
\end{aligned}
$$

and note that these curves are separating.

Theorem 2.4. There is a $K_{2}$ such that, if $k>K_{2}$, then the separating curves $\gamma(p / q+)$ and $\gamma(p / q-)$ defined as above are uniform Lipschitz graphs over $x$ (with Lipschitz constant $\left.1+k \max \left|V^{\prime \prime}(x)\right|\right)$.

Proof. It suffices to do the construction of $\gamma(p / q+)$ only. We will therefore drop the subscript "+" from $W^{u}$ and $W^{s}$. 
For $k>K_{h}(T), D f$ maps the cone field $C$ defined as the set of all tangent vectors $(\alpha, \beta)$ such that $\alpha \beta>0$ strictly into itself for all $x \in I_{-}(k, T)$ (Goroff 1985$)$. So let $T=1$ and let $K_{2}=\max \left\{K_{1}(1), K_{h}(1)\right\}$, the local unstable manifold to $E_{p / q}$ contained in $\bigcup_{i \leqq 0} W^{u}\left(G_{i}\right)$ lies on a Lipschitz graph

$$
g(x)=(x, y(x)) \text { with } \frac{d y}{d x}>0 .
$$

The image under $f$ of such a graph over (a subinterval of) $I_{-}$is again a graph with

$$
\frac{d y_{n+1}}{d x_{n+1}}=\frac{k V^{\prime \prime}\left(x_{n}\right)+d y_{n} / d x_{n}}{1+k V^{\prime \prime}\left(x_{n}\right)+d y_{n} / d x_{n}} .
$$

Since $V^{\prime \prime}$ is bimodal, we have $V^{\prime \prime}\left(x \in I_{-}\right)>0$. Therefore, the Lipschitz constant of the graph satisfies $L \leqq 1$. So $\bigcup_{i \leqq 0} W^{u}\left(G_{i}\right)$ lies on a Lipschitz graph.

The complement of the cone field $C$ is mapped into itself by $D f^{-1}$ [see Eq. (1.1)] as long as $x_{n-1}=x_{n}-y_{n} \in I_{-}$. In fact, the cone field $C=\{(-\alpha, \beta) \mid \beta>\alpha>0\}$ is mapped into itself by $D f^{-1}$ for $x_{n-1} \in I_{-}$. Therefore, local stable manifolds in $\bigcup_{i>0} W^{s}\left(G_{i}\right)$ whose inverse images (under $f$ ) lie in $I_{-}$form a graph $(x, y(x))$ with

$$
\frac{d y_{n}}{d x_{n}}<-1
$$

The image under $f^{-1}$ of such a graph satisfies:

$$
\frac{d y_{n-1}}{d x_{n-1}}=-k V^{\prime \prime}\left(x_{n}-y_{n}\right)+\frac{d y_{n} / d x_{n}}{1-d y_{n} / d x_{n}},
$$

so that

$$
-k V^{\prime \prime}\left(x_{n}-y_{n}\right)-1<\frac{d y_{n-1}}{d x_{n-1}}<-k V^{\prime \prime}\left(x_{n}-y_{n}\right)<-1
$$

Since $V^{\prime \prime}\left(x_{n-1}\right)>0$ if $x_{n-1}=x_{n}-y_{n} \in I_{-}$, this proves that $\bigcup_{i>0} W^{s}\left(G_{i}\right)$ is uniformly Lipschitz (with Lipschitz constant $\left.1+\mathrm{k} \max \left|V^{\prime \prime}(x)\right|\right)$. Furthermore, using (2.1) once more, for $n=1$, one concludes that $d y_{0} / d x_{0}$ is bounded. Therefore, $\bigcup_{i \geqq 0} W^{s}\left(G_{i}\right)$ is a graph.

The following corollary will be instrumental in proving the Single Intersection theorem.

Corollary 2.5. For $k>K_{2}(T)$, the projection of any orbit in $\bigcup_{i \in Z}\left\{W_{+}^{s}\left(G_{i}\right) \cap W_{+}^{u}\left(G_{i}\right)\right\}$ or $\bigcup_{i \in Z}\left\{W_{-}^{s}\left(G_{i}\right) \cap W_{-}^{u}\left(G_{i}\right)\right\}$ visits $I_{+}$only once.

Corollary 2.6. All orbits in $\bigcup_{i \in Z}\left\{W_{+}^{s}\left(G_{i}\right) \cap W_{+}^{u}\left(G_{i}\right)\right\}$ and all orbits in $\bigcup_{i \in Z}\left\{W_{-}^{s}\left(G_{i}\right)\right.$ $\left.\cap W_{-}^{u}\left(G_{i}\right)\right\}$ have type not greater than 1 (in fact, they are well-ordered).

Proof. These orbits lie in $\gamma(p / q+)$ and $\gamma(p / q-)$. As before, we consider only the former case and drop the subscript " + ". Every orbit must have a point, say $(x, y)$, in 
common with $W^{s}\left(G_{0}\right)$ and with $W^{u}\left(G_{0}\right)$. Since $\bigcup_{i \geqq 0} W^{s}\left(G_{i}\right)$ lies on a forward invariant graph and $\bigcup_{i \leqq 0} W^{u}\left(G_{i}\right)$ on a backward invariant graph, it follows that the entire orbit of $(x, y)$ is well-ordered.

\section{Periodic Orbits}

For $k$ sufficiently big, we set up the symbolic dynamics for periodic orbits of bounded type. We prove that there is a one to one correspondence between allowed symbol sequences and well-ordered orbits. Moreover, all these orbits are hyperbolic.

According to Lemma 2.3, the projection of this set $\pi\left(O_{T}\right)$ is contained in $I_{-} U I_{+}+Z$. Consider an orbit of type $t$ in this set with projection $\left\{x_{i}\right\}_{-\infty}^{+\infty}$. Let $X_{i}$ be the unique critical point of $V(x)$ in the interval that $x_{i}$ belongs to. We will call $\left\{X_{i}\right\}$ the symbol sequence of the orbit $\left\{\left(x_{i}, y_{i}\right)\right\}_{-\infty}^{+\infty}$. Define

$$
s_{i}=-2 X_{i}+X_{i-1}+X_{i+1} \text {. }
$$

The type of such an orbit is related to $\left\{s_{i}\right\}$ by

$$
\left|t-\max _{i \in Z}\right| s_{i}||<\delta_{I}
$$

where

$$
\delta_{I}=2 \max \left\{\text { length }\left(I_{-}\right) \text {, length }\left(I_{+}\right)\right\} .
$$

Theorem 3.1. For each $T$ there is a $K_{3}(T)$ such that for each $k>K_{3}(T)$, the following holds: i) to each periodic orbit with type smaller than $T$ corresponds a unique periodic symbol sequence with type smaller than $T+\delta_{I}$, and ii) to each periodic symbol sequence with type smaller than $T$ corresponds a unique periodic orbit with type smaller than $T+\delta_{I}$.

Proof. From the foregoing remarks it is obvious that i) is implied by Lemma 2.3.i.

Let $\left\{X_{i}\right\}$ be an $q$-periodic sequence of type $t^{\prime}$ corresponding to a rotation number $p / q$. Equations (2.1) for the critical point of $H$ can be rewritten in vector form as:

$$
\left(\begin{array}{rrrrrrr}
2 & -1 & 0 & . & . & 0 & -1 \\
-1 & 2 & -1 & . & . & 0 & 0 \\
\cdot & . & . & . & . & . & . \\
. & . & . & 0 & -1 & 2 & -1 \\
-1 & 0 & . & . & 0 & -1 & 2
\end{array}\right) \cdot\left(\begin{array}{c}
x_{1} \\
\vdots \\
x_{q}
\end{array}\right)+k\left(\begin{array}{c}
V^{\prime}\left(x_{1}\right) \\
\vdots \\
V^{\prime}\left(x_{q}\right)
\end{array}\right)=\left(\begin{array}{c}
-p \\
0 \\
\cdot \\
0 \\
p
\end{array}\right) .
$$

Now, define

$$
x_{i}=X_{i}+\xi_{i} .
$$

From the assumptions on $V(x)$ in the introduction, we may write:

$$
\begin{array}{lll}
V^{\prime}\left(X_{i}+\xi_{i}\right)=\xi_{i}-v_{+}(\xi) & \text { if } & X_{i} \in I_{-}+Z, \\
V^{\prime}\left(X_{i}+\xi_{i}\right)=-\xi_{i}+v_{-}(\xi) & \text { if } & X_{i} \in I_{+}+Z,
\end{array}
$$


where $\xi_{i} \in R, v_{-}$and $v_{+}$are twice differentiable, and

$$
|v(\xi)|=O\left(|\xi|_{m}^{2}\right)
$$

[The sign convention is chosen so that $\operatorname{sg}(v(\xi))=\operatorname{sg}(\xi)$ for the standard map.] Now Eq. (3.3) becomes:

$$
\begin{gathered}
\left(\begin{array}{cccccc}
2+\varepsilon_{1} k & -1 & 0 & . & 0 & -1 \\
-1 & 2+\varepsilon_{2} k & -1 & . & 0 & 0 \\
\cdot & \cdot & \cdot & \cdot & \cdot & \cdot \\
\cdot & \cdot & 0 & -1 & 2+\varepsilon_{q-1} k & -1 \\
-1 & 0 & . & 0 & -1 & 2+\varepsilon_{q} k
\end{array}\right) \cdot\left(\begin{array}{c}
\xi_{1} \\
\vdots \\
\xi_{q}
\end{array}\right) \\
-k\left(\begin{array}{c}
v_{1}\left(\xi_{1}\right) \\
\vdots \\
v_{q}\left(\xi_{q}\right)
\end{array}\right)=\left(\begin{array}{c}
s_{1} \\
\vdots \\
s_{q}
\end{array}\right),
\end{gathered}
$$

where

$$
\begin{array}{lllll}
v_{i}=v_{+} & \text {and } & \varepsilon_{i}=+1 & \text { if } & X_{i} \in I_{-}+Z \\
v_{i}=v_{-} & \text {and } & \varepsilon_{i}=-1 & \text { if } & X_{i} \in I_{+}+Z .
\end{array}
$$

Let $L_{q}$ be the Hessian of $H(1.1 \mathrm{c})$ in the points $X_{i}$, then with the obvious notation (3.4a) can be written as:

$$
L_{q} \xi-k v(\xi)=s
$$

Corollary A.5 asserts that $\left(K_{3}(T)>4\right)$ the eigenvalues of $L_{q}$ are greater than $|k-4|$ so that $L_{q}$ is invertible. Define

$$
\psi(\xi)=\left(k L_{q}^{-1}\right)\left(\frac{s}{k}+v(\xi)\right) .
$$

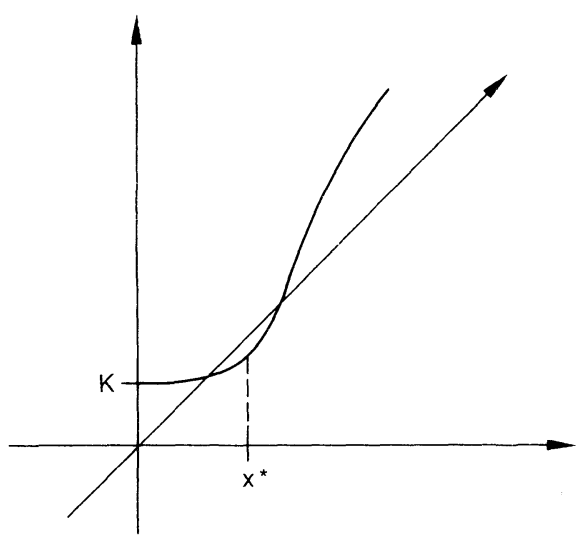

Fig. 3.1. Graph of $f(x)=K+C x^{2}$. At $x=x^{*}$, the derivative of $f$ is $l$. For $\gamma<x^{*}, f(x)<x$ if $K$ small 
We now observe that Theorem B.4 implies that

$$
\|D \psi\|_{m} \leqq\left\|k L_{q}^{-1}\right\|_{m}\left|v^{\prime}(\xi)\right|_{m} \leqq \frac{k}{k-4} 2 C|\xi|_{m}
$$

Thus $\psi$ is a contraction (see Fig. 3.1) on the region $\Delta=[-\delta, \delta]^{q}$ contained in $R^{q}$ if

$$
\begin{gathered}
0<\delta<\gamma<\frac{k-4}{2 k C} \\
|\psi(\xi)|_{m} \leqq\left|L_{q}^{-1} S\right|_{m}+\left\|k L_{q}^{-1}\right\|_{m}|\nu(\xi)|_{m} \leqq \frac{t^{\prime}}{k-4}+\frac{k}{k-4} C|\xi|_{m}^{2} \leqq|\xi|_{m},
\end{gathered}
$$

for $k$ large enough. Thus the region $\Delta$ is mapped into itself. Therefore $\psi$ has a unique fixed point in $\Delta$. By construction, this fixed point corresponds to a unique orbit $\left\{x_{i}\right\}$ of type $t$ with $\left|t-t^{\prime}\right|<\delta_{I}$.

Proposition 3.2. For $k>K_{3}(T)+1$, periodic orbits in $O_{T}$ are hyperbolic.

Proof. We use an identity proved by Greene (1979):

$$
\left|\operatorname{Tr} D f^{q}\right|=|\operatorname{det} \operatorname{Hess}(H)+2|,
$$

where

$$
H=\sum_{i=1}^{q} h\left(x_{i}, x_{i+1}\right)
$$

and

$$
x_{0}=x_{q} \text { and } x_{1}=x_{q+1} .
$$

In the proof of the remark that goes with Proposition A.4 we prove that $\lambda_{i}=2+k V^{\prime \prime}\left(x_{i}\right)$. So, for $\left|2+k V^{\prime \prime}\left(x_{i}\right)\right|>3$, we have

$$
\left|\operatorname{Tr} D f^{q}\right| \geqq \prod_{i=1}^{q}\left(\left|\lambda_{i}\right|-1\right)>2 \text {. }
$$

We note in passing that this does not imply that all periodic orbits in the map are hyperbolic. On the contrary, elliptic orbits can readily be found for arbitrarily large $k$ (see, for instance, Leage and MacKay 1986). However, they have to have type bigger than $T$ in the proposition.

Corollary 3.3. Let o be a periodic orbit in $O_{T}$, and let $k>K_{3}(T)$. Let its Hessian be denoted by $H$. Then the number of negative eigenvalues of $H$ equals the number of points of o that project to $I_{+}$. The other points project to $I_{-}$.

Proof. This is an application of Theorem A.7 and of Theorem 3.1.

\section{Homoclinic Orbits}

By using the same technique as in the previous section, we now include points in $\bigcup_{i=-\infty}^{+\infty}\left\{W^{u}\left(G_{i}\right) \cap W^{s}\left(G_{i}\right)\right\}$ (as defined in Sect. 2) in the symbolic dynamics. Since these orbits are not periodic, we have to adapt the theory of the previous section. We do this here for homoclinic orbits. The result is that other than the endpoints $W^{u}\left(G_{i}\right)$ 
$\cap W^{s}\left(G_{i}\right)$ contains a single point. We will make use of the constants $K_{i}(T)$ defined in the previous section.

We will make use of the following notation. A homoclinic m-advancing orbit $\left\{x_{i}\right\}$ to an orbit $\left\{z_{i}\right\}$ (not necessarily well-ordered) with $z_{i+q}=z_{i}+p$ (here $p$ and $q$ are not necessarily relative primes) is an orbit such that

$$
\lim _{k} x_{-k q}+k p=z_{0}, \quad \lim _{n} x_{k q-m}-k p=z_{0} .
$$

Lemma 2.3 implies that if its type is small enough it corresponds to a unique symbol sequence $\left\{X_{i}\right\}$ with there is an $N$ such that

$$
\begin{aligned}
& \text { if } i>N \quad X_{i}=Z_{i+m} \\
& \text { if } \quad i<-N \quad X_{i}=Z_{i} \text {. }
\end{aligned}
$$

The symbol sequence $\left\{Z_{i}\right\}$ is also called $m$-advancing. One defines receding orbits in a similar fashion. Such an orbit is, for all $n$, a critical point of

$$
H_{n}=\sum_{i=-n q+1}^{n q-m} h\left(x_{i}, x_{i+1}\right)
$$

with endpoints fixed. Set $x_{i}=X_{i}+\xi_{i}$. With the same conventions as in Sect. 3, the equations for a critical point become:

$$
M_{n, q} \xi-k v_{n}(\xi)=s_{n}+\eta_{n} .
$$

The vector $\left(\begin{array}{c}\xi_{-n q} \\ 0 \\ \cdot \\ 0 \\ \xi_{n q-m+1}\end{array}\right)$ is denoted by $\eta_{n}$. The matrix $M_{n q}$ has size $2 n q-m$, and has entries equal to zero in the upper right and lower left corners (corresponding to the endpoints being fixed). Equation (4.2a) is equivalent with

where $\mu_{n}$ is the vector $\left(\begin{array}{c}L_{n q} \xi-k v_{n}(\xi)=s_{n}+\mu_{n}, \\ \xi_{-n q}-\xi_{n q-m} \\ 0 \\ \cdot \\ 0 \\ \xi_{n q-m+1}-\xi_{-n q+1}\end{array}\right)$. By $\xi^{*}$ we will denote the solution of the "periodic" problem with symbolic sequence $\left\{X_{i}\right\}_{i=-n q+1}^{n q-m}$ (see Sect. 3):

$$
L_{n q} \xi^{*}-k v_{n}\left(\xi^{*}\right)=s_{n} \text {. }
$$

We say that the limit of a sequence of vectors $\{\xi(i)\}$ exists if the components converge (i.e.: $\lim _{i \rightarrow \infty}(\xi(i))_{p}$ exists for $p$ fixed).

Theorem 4.1. For each $T$ there is a $K_{3}(T)$ such that for each $k>K_{3}(T)$, the following holds: i) to each homoclinic orbit with type smaller than $T$ corresponds a unique asymptotically periodic symbol sequence with type smaller than $T+\delta_{I}\left(\delta_{I}\right.$ as 
defined in (3.2)), and ii) to each asymptotically periodic symbol sequence with type smaller than $T$ corresponds a unique asymptotically periodic orbit with type smaller than $T+\delta_{I}$.

Proof. As in Theorem 3.1, if $k$ is large enough Lemma 2.3 implies that each orbit $\left\{x_{i}\right\}$ corresponds to a unique sequence $\left\{X_{i}\right\}$ (which proves i)).

To prove ii) we may assume that we have an $m$-advancing asymptotically $q$-periodic sequence $\left\{X_{i}\right\}$. We have to prove that there is a unique orbit $\left\{x_{i}\right\}$ (with symbolic sequence $\left\{X_{i}\right\}$ ) homoclinic to a periodic orbit $\left\{z_{i}\right\}_{-\infty}^{+\infty}$.

We first show existence of a homoclinic orbit with the given sequence. Consider the periodic orbit corresponding the symbolic sequence $\left\{X_{i}\right\}_{i=-n q+1}^{n q-m}$. Call the corresponding solution of Eq. (4.2c) $\xi^{*}(n)$. Since according to Theorem 3.1 the components of $\xi^{*}(n)$ are bounded by $\delta_{I}$, limit-points of the sequence $\left\{\xi^{*}(n)\right\}$ exist. Such a limit-point corresponds to a homoclinic orbit with the required symbolic sequence.

We next show uniqueness of the above limit-point. Let $\xi(n)$ be the solution of (4.2b) [which is equivalent to (4.2a)], where we have the freedom to choose $\left|\mu_{n}\right|_{m}<2 \delta_{I}$. Recall that the only non-zero components of $\mu_{n}$ are the first and the last. To see that the limit-point is unique, one observes that the vector $\left((\xi(n+r))_{-n q+1}, \ldots,(\xi(n+r))_{n q-m}\right)$ satisfies Eq. (4.2b) for some bounded $\mu_{n}$. On the other hand, $\left((\xi(n+r))_{-n q+1}, \ldots,(\xi(n+r))_{n q-m}\right)$ satisfies Eq. (4.2b) with $\mu_{n}=0$. Thus Lemma B.2 implies that $\xi(n)$ is a Cauchy sequence.

Remark. The existence of well-ordered advancing and receding orbits has been proved by Katok $(1982 \mathrm{a}, \mathrm{b})$ in a more general context.

Theorem 4.2 ("Single Intersection"). For all $k>\max \left\{K_{2}, K_{3}(1)\right\}$, we have that $\Lambda=\bigcup_{i=-\infty}^{+\infty}\left\{W^{u}\left(G_{i}\right) \cap W^{s}\left(G_{i}\right)\right\}$ contains one minimum and one minimax and no other orbit.

Proof. A homoclinic minimax $\left\{x_{i}\right\}$ is an orbit such that for all $n$ big enough the Hessian $D^{2} H_{n}$ has a single negative eigenvalue. By Theorem A.7 (and the fact that the norm of $v_{n}$ is small), this implies that one diagonal term in the Hessian (4.2a) is negative, or, equivalently, that there is a unique $i$ such that $X_{i} \in I_{+} \cup Z$. (A minimum has only positive eigenvalues.) Thus existence and uniqueness of the 1 -advancing well-ordered minimum and minimax follow from the previous proposition. (These orbits have type not exceeding 1.)

From Corollary 2.6, one concludes that all orbits in $\Lambda$ have type not greater than one. Suppose now that there is an orbit in $\Lambda$ for which the Hessian in the previous proposition has more than one negative eigenvalue. Theorem A.7 applies again. (Strictly speaking, we have to modify the theorem, because the anti-diagonal elements in the matrix vanish. But that is as straightforward as it was in the proof of the proposition.) Thus the alleged homoclinic orbit $\left\{x_{i}\right\}$ lands at least two times in $I_{+}$. But this contradicts Corollary 2.5.

Remark. This theorem (and other results relying on hyperbolicity and uniqueness of orbits with bounded type) generalizes to irrational rotation numbers. The generalization of Eq. (4.2) is given in the proof of Proposition 5.3. 


\section{Symmetry Lines and Diamonds}

In this section, we prove that for the standard map each minimax orbit, whether periodic or homoclinic, has a point in common with a reflection line. In addition, we prove some results concerning the topology of Aubry Mather sets. These latter ones were conjectured to hold for all $k$ (Veerman and Tangerman 1988a).

Define

$$
S:\left\{\begin{array}{l}
x \rightarrow-x \\
y \rightarrow y+k V^{\prime}(x) .
\end{array}\right.
$$

Suppose further that $V^{\prime}(x)=-V^{\prime}(-x)$ and that $x=0$ is contained in $I_{+}$(the latter can be achieved by conjugating by a translation in the $x$-direction). An example of a map that satisfies these requirements is the standard-map. Then $x=0$ is a socalled symmetry line (in the example of Sect. 6 , we have the symmetry line at $x=1 / 2$ ). That is: $S$ satisfies

$$
S f S=f^{-1}
$$

and

$$
S^{2}=\mathrm{Id}
$$

and it leaves the line $x=0$ invariant (Greene 1979). In this case, we have the following theorem.

Theorem 5.1. For $k>K_{3}(T)$, we have that the projection of an orbit in $O_{T}$ with symmetric symbol sequence is invariant under reflection in $x=0$.

Proof. We start with the observation that $f^{-1}$ is generated by the action $h\left(x^{\prime}, x\right)$ as remarked in the introduction. Therefore, if $\left\{x_{i}\right\}_{-\infty}^{+\infty}$ is a critical point of $H=\sum_{i=-\infty}^{+\infty} h\left(x_{i}, x_{i+1}\right)$ with rotation number $\varrho$, then $\left\{x_{-i}\right\}_{-\infty}^{+\infty}$ is a critical point of $H^{\prime}=\sum_{i=-\infty}^{\infty} h\left(x_{i+1}, x_{i}\right)$ with the same rotation number. Further, if $\left\{x_{i}\right\}$ is a critical point of $H$ (orbit of $f$ ) and has a symmetric symbol sequence, then, by reflection, $\left\{-x_{i}\right\}=\left\{\pi S\left(x_{i}, y_{i}\right)\right\}$ is a critical point of $H^{\prime}$ (orbit of $f^{-1}$ ) and the same symbol sequence as $\left\{x_{-i}\right\}$. But by uniqueness of these orbits, it follows that $\left\{x_{i}\right\}$ must be invariant under reflection $\left(x_{-i}=-x_{i}\right)$.

Corollary 5.2. For $k>K_{3}(1)$, each order preserving minimax orbit has a point in common with the symmetry line.

Proof. A minimax has only one point in $I_{+}$(see Theorem A.7). Call this point $x_{0}$. Order preserving implies that the symbol sequence is symmetric. The result now follows from the previous theorem.

Remark. The fact that minimum orbits have no point in common with the line $x=0$, is, of course, directly implied by Lemma $2.3 \mathrm{ii}$ (for large $k$ ).

Proposition 5.3. Let $x$ be a limit-point of minimizing periodic points such that its orbit does not have a rational rotation number. For $k>K(1)$, its orbit is recurrent.

Proof. Since the proof is very similar to that of Theorem 4.1, we only give an outline of the proof. We know that any orbit contained in the limit $f$ minimizing well- 
ordered orbits is again minimizing and well-ordered (Mather 1986). Such an orbit must therefore have a rotation number which, by assumption, is irrational. Its $\omega$-limit set $E_{\rho}$ is a recurrent set. Suppose that the orbit $\left\{x_{i}^{*}\right\}_{-\infty}^{+\infty}$ of an orbit constructed that way is non-recurrent. Such an orbit must live in a gap in $E_{\varrho}$ and its symbol sequence $\left\{X_{i}^{*}\right\}_{-\infty}^{+\infty}$ is identical to the sequence $\left\{X_{i}\right\}_{-\infty}^{+\infty}$ of either the left- or the right endpoint of the gap. Furthermore, both endpoints are bi-asymptotic to each other. So, we can set up an operator $L_{q_{n}}$ (where $q_{n}$ are, say, the denominators of the convergents to $\varrho$ ) and vectors $s_{q_{n}}$ and $\mu_{q_{n}}$ as in (4.2b) with the property that $\lim _{n}\left|\mu_{q_{n}}\right|=0$. For the solution $\xi^{*}$ living in the gaps, we reach the same conclusion, that is: the orbit is identical to the orbit of the endpoint of the gap.

Now, suppose, that $E_{\alpha}$ is hyperbolic, then by arguments given in Veerman and Fangerman (1990), there is an open interval of rotation numbers $D$ containing $\alpha$, such that $H=\bigcup_{\varrho \in D} E_{\varrho}$ is a hyperbolic set. We can then define $\varepsilon$-local stable and unstable manifolds at each point of $H$ which have bounded diameter greater than $\delta>0$. For the remainder of this section, let $\alpha$ irrational, $r / s>\alpha>p / q$.

Theorem 5.4 ("Diamond Configuration"). If $k>\max \left\{K_{2}, K_{3}(1)\right\}, E_{\alpha}$ is hyperbolic and for $r / s$ and $p / q$ close enough to $\alpha, E_{\alpha}$ is contained in a region $K$ which is the union of "diamonds" and whose boundary is formed by local stable and unstable manifolds to $E_{p / q}$ and $E_{r / s}$ only.

Proof. By Theorem 2.4, $\gamma(r / s-)$ and $\gamma(p / q+)$ can be constructed so that they are Lipschitz graphs. Lipschitz graphs satisfy the hypothesis of the Monotonicity theorem (Condition 2.4 in Veerman and Tangerman 1990). This theorem then states (Veerman and Tangerman 1990) that if $K$ is the open set defined by:

$$
\begin{aligned}
& K \text { is "below" } \gamma(r / s-), \\
& K \text { is "above" } \gamma(p / q+)
\end{aligned}
$$

(here "above" and "below" have their usual meaning since $\gamma(p / q+)$ and $\gamma(r / s-)$ are Jordan curves), then $E_{\alpha}$ is contained in $K$.

Assume that $x \in E_{\alpha}$ lies in a region not bounded by local invariant manifolds (see Fig. 5.1). Then there must be a stable segment that intersects an unstable segment

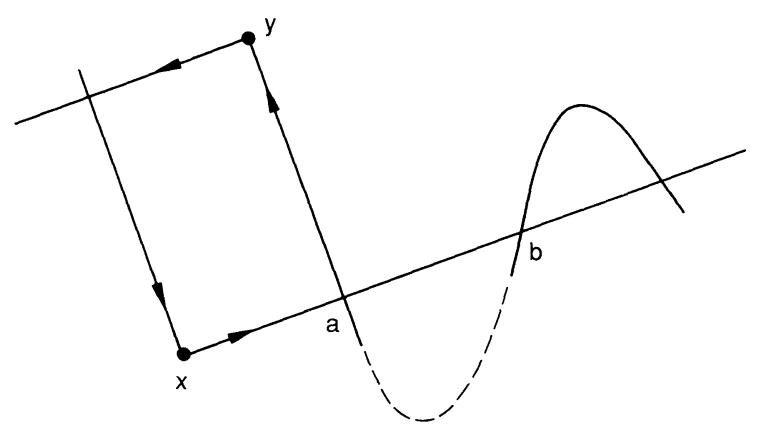

Fig. 5.1. $W^{s}(y)$ intersects $W^{u}(x)$ in the points a and b 
more than once. But that is in contradiction with the fact that stable segments have slope less than -1 while all unstable segments have slope between 0 and 1 .

\section{Estimates for the Standard Map}

In order to form some idea about the size of the constants $K_{i}$ for which the most important conclusions hold, we evaluate $K_{3}(1)$, needed in Theorems 3.1, 5.1, and 5.4 as well as Theorem 4.1, for the standard map $\left(\right.$ i.e.: $\left.V^{\prime}(x)=\frac{\sin 2 \pi x}{2 \pi}\right)$. We limit these considerations exclusively to minima and minimaxes.

In Sect. 2, one immediately has from Lemma 2.3 that

$$
\begin{gathered}
K_{1}(T)=2 \pi T . \\
x \in I_{-}(k, T) \Leftrightarrow|\sin 2 \pi x| \leqq \frac{2 \pi T}{k}, \quad x<1 / 4,
\end{gathered}
$$

and

$$
x \in I_{+}(k, T) \Leftrightarrow|\sin 2 \pi x| \leqq \frac{2 \pi T}{k}, \quad|x-1 / 2|<1 / 4
$$

Further, we have from Theorem 2.4 and from Goroff (1985) that

$$
K_{2}=\max \left\{K_{1}(1), K_{h}(1)\right\}=K_{h}(1)=2\left(1+\pi^{2}\right)^{1 / 2} .
$$

The constant $K_{3}(1)$ in Sect. 3 is slightly more problematic. From the proof of Theorem 3.1, we obtain that $K_{3}(T)$ must be greater than $T+3$ (Theorem B.4), greater than 4 (Theorem A.5), and big enough for Eq. (3.5) to define a contraction. In the reasoning below, we verify that (3.5) defines a contraction for $k>2 \pi$.

Lemma 6.1. For order preserving minimizing and minimax orbits (periodic and homoclinic) $\left\|k L^{-1}\right\| \leqq 1$.

Proof. For minimizing orbits, the results follows from Theorems B.4 and B.5. For minimax orbits, there is a unique $I \in N$ such that $\lambda_{I}<0$ and $\xi_{I}=0$ (Corollary 5.2).

Proposition B.3 is now still valid with $\min _{i}$ replaced by $\underset{i \neq I}{\min }$ and so are its Corollaries B.4 and B.5.

Theorem 6.2. In the case of the standard map $K_{3}(1)$ equals $2 \pi$.

Proof. We have (see proof of Theorem 3.1)

$$
|v(\xi)|_{m}=|\xi|_{m}-\frac{1}{2 \pi} \sin \left(2 \pi|\xi|_{m}\right)
$$

It is sufficient to prove that for $k>2 \pi$ the map $\psi$

$$
\psi: \xi \rightarrow\left(k L^{-1}\right)\left(\frac{s}{k}+v(\xi)\right)
$$

is contracting on any region $\Delta_{\gamma}$ defined by $|\xi|_{m}<\gamma<1 / 4$ and maps that region into itself. 
Since

$$
\left|\psi^{\prime}(\xi)\right|_{m} \leqq\left\|k L^{-1}\right\|_{m}\left|v^{\prime}(\xi)\right|_{m} \leqq 1-\cos \left(2 \pi|\xi|_{m}\right)
$$

$\psi$ is indeed a contraction on $\Delta_{\gamma}$ for $\gamma<1 / 4$. In addition,

$$
|\psi(\xi)|_{m} \leqq\left\|k L^{-1}\right\|\left(\frac{|s|_{m}}{k}+|v(\xi)|_{m}\right)=\frac{1}{k}+|\xi|_{m}-\frac{1}{2 \pi} \sin \left(2 \pi|\xi|_{m}\right)
$$

The graph of $\frac{1}{k}+x-\frac{1}{2 \pi} \sin 2 \pi x$ (see Fig. 3.1) is increasing and intersects the diagonal only if $k \geqq 2 \pi$. If $k \geqq 2 \pi, \Delta_{\gamma}$ is mapped into itself for $\gamma<1 / 4$ and close to $1 / 4$.

\section{Concluding Remarks}

While our results are valid only for large $k$, we believe that an extension of these results should be valid for all $k$. Here is some of the evidence. The Single Intersection property is observed for all $k$, and can also be proven by perturbative techniques (Simo, personal communication) for small $k$ (though not uniform in the rotation number). The hyperbolicity of Aubry Mather sets (which is an essential ingredient in this work) is observed numerically as soon as the invariant curve has broken up ( $\mathrm{Li}$ and Bak 1986). We expect that the structure of the invariant manifolds that we outlined in Theorem 2.4 is very regular for all $k$ : it is not necessarily uniformly Lipschitz, but it will probably consist of finitely many graphs, which may be sufficient to prove many of the results of this and previous works. The way to achieve such results might be to try to prove them for high iterates of the map we considered in this paper and apply renormalization arguments.

We expect that some of the results concerning stable and unstable manifolds discussed in this and previous works also generalize to the dissipative standard map. It is easy to extend Eq. (2.1) to that case (just multiply the first term by the dissipation). One could then paraphrase the reasoning in the appendices of this paper by considering a system of such equations (bearing in mind, of course, that in this case not all rotation numbers are realized). The result concerning Convergence of Turnstiles in Veerman and Tangerman (1990) would provide us with detailed knowledge as to how unstable manifolds pile up in this case.

It is also possible that an extension of this essentially linear construction generalizes to four-dimensional symplectic maps, such as the ones studied by Angenent (1988) or Kook and Meiss (1988) and requiring, again, that the necessary nonlinearity parameter is sufficiently big. This way, one should be able to establish the existence and the structure of such Cantor sets, in the way that is done in Veerman and Tangerman (1989) and in Tangerman and Veerman (1990).

Finally, it should be noted that the uniform estimates on the periodic orbits achieved in Sect. 3, enable one to take Hausdorff limits of such sets and construct invariant Cantor sets for a given rotation number that have a prescribed fraction of points in $I_{+}$(that is: the Hessian has a certain fraction of its eigenvalues negative). 


\section{Appendix A}

In this appendix, we analyze the eigenvalues of the Hessian matrix for large $k$. The matrices we are interested in are those of Eqs. (3.4a) and (4.2a).

Let

$$
L_{q}=\left(\begin{array}{rrrrcc}
\lambda_{1} & -1 & 0 & . & 0 & -\delta \\
-1 & \lambda_{2} & -1 & . & 0 & 0 \\
\cdot & . & . & . & . & . \\
\cdot & . & 0 & -1 & \lambda_{q-1} & -1 \\
-\delta & 0 & . & 0 & -1 & \lambda_{q}
\end{array}\right)
$$

where $\delta=1$. Denote the $n \times n(n<q)$ upper left matrix by $M_{n}$ and its determinant by $\Delta_{n}$. The above matrix with $\delta=0$ will be denoted by $M_{q}$ and its determinant by $\Delta_{q}$. For $n \leqq q$, denote the matrix obtained by deleting the first row and the first column from $\bar{M}_{n}$ by $M_{n}^{*}$ and its determinant by $\Delta_{n}^{*}$.

The following recursive relations are immediate:

Lemma A.1. If $\Delta_{-1}=0, \Delta_{0}=1, \Delta_{0}^{*}=0, \Delta_{1}^{*}=1$, and $\Delta_{-1}^{*}=1$, then, for $i \geqq 1$,

$$
\Delta_{i}=\lambda_{i} \Delta_{i-1}-\Delta_{i-2} \text {, }
$$

and

$$
\Delta_{i}^{*}=\lambda_{i} \Delta_{i-1}^{*}-\Delta_{i-2}^{*},
$$

where

$$
\operatorname{det}\left(L_{q}\right)=\Delta_{q}-\Delta_{q-1}^{*}-2=\lambda_{q} \Delta_{q-1}-\Delta_{q-1}^{*}-\Delta_{q-2}-2 .
$$

Proof. Expand the determinant on the last row.

In the remainder of this appendix we will assume that $\left|\lambda_{i}\right|>2$.

Lemma A.2. $\left|\Delta_{i}\right| \geqq\left(\left|\lambda_{i}\right|-1\right)\left|\Delta_{i-1}\right|,\left|\Delta_{i}^{*}\right| \geqq\left(\left|\lambda_{i}\right|-1\right)\left|\Delta_{i-1}^{*}\right|$, and $\left|\Delta_{i}\right| \geqq\left(\left|\lambda_{1}\right|-1\right)\left|\Delta_{i}^{*}\right|$.

Proof. For $\Delta_{i}$ we have from Lemma A.1:

$$
\left|\frac{\Delta_{i}}{\Delta_{i-1}}\right|=\left|\lambda_{i}-\frac{\Delta_{i-2}}{\Delta_{i-1}}\right|
$$

and the first statement is checked by induction. Similarly for $\Delta_{i}^{*}$. The last statement follows by symmetry.

Lemma A.3. For all $i,|a| \geqq 1,\left|a \Delta_{i}-\Delta_{i}^{*}\right| \geqq\left(\left|\lambda_{i}\right|-1\right)\left|a \Delta_{i-1}-\Delta_{i-1}^{*}\right|$.

Proof. From Lemma A.1, we obtain

$$
\left|\frac{a \Delta_{i}-\Delta_{i}^{*}}{a \Delta_{i-1}-\Delta_{i-1}^{*}}\right|=\left|\lambda_{i}-\frac{a \Delta_{i-2}-\Delta_{i-2}^{*}}{a \Delta_{i-1}-\Delta_{i-1}^{*}}\right| .
$$

For $i=1$, one easily checks that this statement holds and one continues again by induction.

Proposition A.4. $\operatorname{det}\left(L_{q}\right) \neq 0$. 
Proof. First suppose that at least one $\lambda_{i}$ is negative. From the last equation of Lemma A.1:

$$
\left|\operatorname{det}\left(L_{q}\right)+2\right| \geqq\left|\operatorname{sg}\left(\lambda_{q}\right)\left(\left|\lambda_{q}\right|-1\right) \Delta_{q-1}-\Delta_{q-1}^{*}\right| .
$$

By Lemma A.2, the first term in the right-hand side dominates the other two. Thus with $\left|\Delta_{q-2}\right|$ smaller $\left|\Delta_{q-1}\right|$ :

$$
\left|\operatorname{det}\left(L_{q}\right)+2\right| \geqq\left|\left(\left|\lambda_{q}\right|-1\right) \Delta_{q-1}-\Delta_{q-1}^{*}\right| .
$$

Now apply Lemma A.3. Suppose $i$ is the smallest integer so that $\lambda_{i}<0$. The corresponding right-hand side in the proof of Lemma A.3 is greater than 3 .

If all $\lambda_{i}$ are greater than two, then from Lemma A.1,

$$
\frac{\left|\Delta_{i}-\Delta_{i-1}^{*}\right|}{\left|\Delta_{i-1}-\Delta_{i-2}^{*}\right|}=\left|\frac{\lambda_{i} \Delta_{i-1}-\lambda_{i-1} \Delta_{i-2}^{*}}{\Delta_{i-1}-\Delta_{i-2}^{*}}-\frac{\Delta_{i-2}-\Delta_{i-3}^{*}}{\Delta_{i-1}-\Delta_{i-2}^{*}}\right|>1 \text {. }
$$

Since

$$
\Delta_{1}-\Delta_{0}^{*}=\lambda_{1}>2
$$

it follows (by induction) that

$$
\Delta_{q}-\Delta_{q-1}^{*}>2 \text {. }
$$

From Lemma A.1 we obtain that $\left|\operatorname{det}\left(L_{q}\right)+2\right|>2$. Therefore

$$
\operatorname{det}\left(L_{q}\right) \neq 0 \text {. }
$$

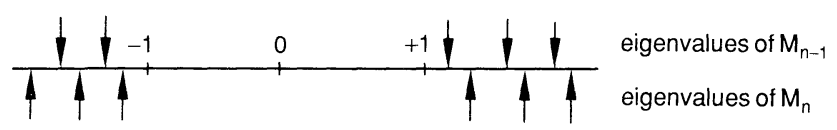

Fig. A.1. The eigenvalues of $M_{n}$ and $M_{n-l}$

Corollary A.5. If $\mu$ is an eigenvalue of the Hessian then there is an isuch that

$$
\left|\lambda_{i}-\mu\right|<2 \text {. }
$$

Proof. If not, then the previous proposition gives

$$
\operatorname{det}\left(L_{q}-\mu I\right) \neq 0 \text {. }
$$

Lemma A.6. If $\left|\lambda_{i}\right|>3$,

$$
\begin{gathered}
\operatorname{sg}\left\{\Delta_{i} / \Delta_{i-1}\right\}=\operatorname{sg}\left\{\lambda_{i}\right\}, \\
\operatorname{sg}\left\{\operatorname{det}\left(L_{q}\right) / \Delta_{q-1}\right\}=\operatorname{sg}\left\{\lambda_{q}\right\}, \text { for } q>2 .
\end{gathered}
$$

Proof. The first relation follows immediately from Lemmas A.1 and A.2. For the second relation:

$$
\frac{\operatorname{det}\left(L_{q}\right)}{\Delta_{q-1}}=\lambda_{q}-\frac{\Delta_{q-1}^{*}}{\Delta_{q-1}}-\frac{\Delta_{q-2}}{\Delta_{q-1}}-\frac{2}{\Delta_{q-1}},
$$

where the individual terms can be estimated by using Lemma A.2. 
Theorem A.7. If $\left|\lambda_{i}\right|>3$, the number of negative eigenvalues (counting multiplicity) of the Hessian $L_{q}$ equals the number of negative terms on the diagonal.

Proof. We will make use of the fact that the matrices considered are symmetric. That implies that they are diagonalizable, have real eigenvalues and that different eigenspaces are orthogonal. Moreover (see Wilf 1978), the eigenvalues of $M_{i-1}$ separate the eigenvalues of $M_{i}$ (and $M_{q-1}$ those of $L_{q}$ ) as depicted in Fig. A.1. (In case $M_{i}$ has an eigenvalue with multiplicity $p$, then $M_{i-1}$ has that eigenvalue with multiplicity $p-1$.)

Let $\mu_{i}^{n}$ denote the $i^{\text {th }}$ eigenvalue of $M_{n}$. All of these eigenvalues lie outside the interval $[-1,+1]$ by Corollary A.5. Knowing the sign of the eigenvalues of $M_{n}$ determines the sign of all but one of the eigenvalues of $M_{n-1}$ (see Fig. A.1). So that sign has to be determined by $\operatorname{sg}\left\{\Delta_{n} / \Delta_{n-1}\right\}$ from which the theorem follows.

\section{Appendix B}

In this appendix, we consider for $k$ sufficiently big, the solutions of the following linearized problems.

The periodic problem:

$$
L_{q} \xi=s
$$

where $\xi=\left(\xi_{1}, \xi_{2}, \ldots, \xi_{q}\right)$ and $|s|_{m} \leqq T$.

- The $m$-advancing homoclinic problem:

For all $n$ :

$$
M_{n, q} \xi=s_{n}+\eta_{n},
$$

where $M_{n, q}=M_{2 n q-m}$ as defined in Appendix A,

$$
\xi=\left(\xi_{-n q+1}, \xi_{-n q+2}, \ldots, \xi_{0}, \xi_{1}, \ldots, \xi_{n q-m}\right),
$$

$\left|\left(s_{n}\right)\right|_{m} \leqq T$, and $\eta_{n}=\left(C_{1}(n), 0,0, \ldots, 0, C_{2}(n)\right)$ with $\left|\eta_{n}\right|_{m} \leqq C$ independent of $n$.

In the last problem the only parameter of interest is $n$. The sequence of vectors $\left\{s_{n}\right\}$ is chosen so that if $k>n$, corresponding components numbered $-n q+1$ to $n q-m$ of the vectors $s_{k}$ and $s_{n}$ are equal. We are interested in the sequence of solutions $\{\xi(n)\}$ to $(\mathrm{B} .1 \mathrm{~b})$.

In both problems the object is to prove that the solutions $\xi$, respectively $\xi(n)$, have components of uniformly bounded size. Furthermore, the sequence of solutions $\{\xi(n)\}$ converges component wise.

Let $\lambda_{i}$ be the diagonal elements of $L_{q}$, respectively $M_{n, q}$, and let $\mu_{i}$ its eigenvalues. Throughout this appendix, we will assume that $\left|\lambda_{i}\right|>T+3$.

Lemma B.1. At least one component of $\xi$ satisfies: $\left|\xi_{i}\right| \leqq \frac{T}{\min _{i}\left|\lambda_{i}\right|-2}$.

Proof. Denote with $d$ the dimension of the space acted on by the operator $L_{q}$ (or $\left.M_{n, q}\right)$. Then:

$$
\sqrt{d}\left(\min _{i}\left|\xi_{i}\right|\right) \leqq|\xi| \leqq \frac{|s|}{\left(\min _{i}\left|\mu_{i}\right|\right)} \leqq \frac{\sqrt{d} T}{\left(\min _{i}\left|\lambda_{i}\right|-2\right)}
$$


(The last inequality follows from Corollary A.5.)

In the next lemma we consider the homoclinic problem. Denote by $\xi^{*}(n)$ the solution to (B.1b) with $\eta_{n}=0$.

Lemma B.2 (Exponential Decay).

$$
\left|\xi(n)_{i}-\xi^{*}(n)_{i}\right| \leqq 2^{-|n q-m-i|}\left|\eta_{n}\right|_{m} .
$$

Proof. Denote the components of $\left(\xi(n)-\xi^{*}(n)\right)$ by $t(n)_{i}=t_{i}$. The above equation implies (except for the components $i=-n q+1$ and $i=n q-m$ ),

$$
\lambda_{i} t_{i}=t_{i-1}+t_{i+1} \text {. }
$$

So if

$$
\left|t_{i}\right|>\frac{1}{2}\left|t_{i-1}\right|
$$

then

$$
\left|\lambda_{i}\right|\left|t_{i}\right| \leqq\left|t_{i-1}\right|+\left|t_{i+1}\right| \leqq 2\left|t_{i}\right|+\left|t_{i+1}\right|
$$

Thus

$$
\left|t_{i+1}\right|>2\left|t_{i}\right| .
$$

This implies that the graph of $\left|t_{i}\right|$ versus $i$ is exponentially increasing (with a factor at least 2 each time) as soon as it is not exponentially decreasing (with a factor at least 2). Now consider the first and the last equations:

$$
\begin{aligned}
\lambda_{-n q+1} t_{-n q+1}-t_{-n q+2} & =C_{1}(n), \\
\lambda_{n q-m} t_{n q-m}-t_{n q-m-1} & =C_{2}(n) .
\end{aligned}
$$

There are now three cases to consider:

- The graph of $\left|t_{i}\right|$ versus $i$ is exponentially increasing with a factor at least 2 . Then we conclude that $\left|t_{n q-m}\right| \leqq C_{2}(n) \leqq\left|\eta_{n}\right|_{m}$.

- The graph of $\left|t_{i}\right|$ versus $i$ is exponentially decreasing with a factor at least 2 . Then we conclude that $\left|t_{-n q+1}\right| \leqq C_{1}(n) \leqq\left|\eta_{n}\right|_{m}$.

- The graph of $\left|t_{i}\right|$ versus $i$ is first exponentially decreasing and subsequently exponentially increasing (with a factor at least 2). In this case we also have that $\left|t_{n q-m}\right| \leqq C_{2}(n) \leqq\left|\eta_{n}\right|_{m}$ and $\left|t_{-n q+1}\right| \leqq C_{1}(n) \leqq\left|\eta_{n}\right|_{m}$.

In all cases we obtain that

$$
\left|t_{i}\right| \leqq 2^{-|n q-m-i|}\left|\eta_{n}\right|_{m} .
$$

Denote the component that satisfies the inequality of Lemma B.1 by $\xi_{I}$.

Proposition B.3. If $\left|\xi_{I}\right|<\frac{T}{\min _{i}\left|\lambda_{i}\right|-2}$, then $\left|\xi_{I+1}\right|<\frac{T}{\min _{i}\left|\lambda_{i}\right|-2}$.

Proof. First we observe that for periodic orbits $\xi_{0}=\xi_{q}$ and for $m$-advancing (or $m$-receding) homoclinic orbits $\xi_{-k q}-\xi_{k q-m}$ converges to zero exponentially fast. The former claim, of course, follows directly from (B.1b) which has periodic boundary conditions. The second can be seen as follows. Since $\xi$ must be a solution of (B.1a) for all $n$, we have that for $n=2 k, \xi_{-k q+i}$ and $\xi_{k q-m+i}$ satisfy the same 
equations for $i \in\{-(k-1) q, \ldots, k q\}$. Therefore, the argument in Lemma B.2 applies and $\xi_{-k q}-\xi_{k q-m}$ must go to zero exponentially fast.

Writing out the equation for $\xi_{i+1}$, one has

$$
\xi_{i+1}=\lambda_{i} \xi_{i}-\xi_{i-1}-s_{i}
$$

We have that if

$$
\left|\xi_{i}\right|>\frac{T}{\min _{i}\left|\lambda_{i}\right|-2} \text { and }\left|\xi_{i}\right|>\left|\xi_{i-1}\right|
$$

then

$$
\left|\frac{\xi_{i-1}+s_{i}}{\xi_{i}}\right|<\left|1+\frac{s_{i}}{T}\left(\min _{i}\left|\lambda_{i}\right|-2\right)\right|<\min _{i}\left|\lambda_{i}\right|-1
$$

So

$$
\left|\frac{\xi_{i+1}}{\xi_{i}}\right|=\left|\lambda_{i}-\frac{\xi_{i-1}+s_{i}}{\xi_{i}}\right|>1
$$

Suppose now that the proposition does not hold. Since by assumption (B.3) holds for $i=I+1$, we obtain that

$$
\left|\xi_{I+2}\right|>\left|\xi_{I+1}\right| \text {. }
$$

By straightforward induction (noting that $\xi_{0}=\xi_{q}$ if the orbit is periodic, or $\left(\xi_{-n q}-\xi_{n q-m}\right)$ is exponentially small if the orbit is homoclinic), one then proves that $\left|\xi_{I}\right|>\frac{T}{\min _{i}\left|\lambda_{i}\right|-2}$, contrary to our earlier assumption.

Theorem B.4. Let $X_{0}=X_{q}+p$ and $X_{1}=X_{q+1}+p$. If the sequence $\left\{X_{i}\right\}_{i=0}^{q+1}$ has type $t \leqq T$, then the linear equations (B.1) with periodic boundary conditions have a unique solution $\xi$ with $\xi_{0}=\xi_{q}$ and $\xi_{1}=\xi_{q+1}$ and $\left|\xi_{i}\right|<\frac{T}{\min _{j}\left|\lambda_{j}\right|-2}$.

Proof. By induction. Suppose $\left|\xi_{I}\right|, \ldots,\left|\xi_{I+n}\right|$ satisfy the inequality. If $\left|\xi_{I+n+1}\right|$ does not satisfy it then by Proposition B.3, $\left|\xi_{I}\right|$ does not satisfy it.

Theorem B.5. Let $\left\{X_{i}\right\}_{i=-\infty}^{+\infty}$ be an asymptotically $q$-periodic $m$-advancing sequence (corresponding to an asymptotically $q$-periodic $m$-advancing orbit) with type $t \leqq T$, then the linear equations (B.1) without periodic boundary conditions have a unique solution $\xi$ and $\left|\xi_{i}\right|<\frac{T}{\min _{j}\left|\lambda_{j}\right|-2}$.

Proof. Now $\xi \in R^{n q-m}$. Lemma B.1 implies that there is at least one component of $\xi$ such that

$$
\left|\xi_{I}\right| \leqq \frac{T}{\min _{i}\left|\lambda_{i}\right|-2}
$$


According to Proposition B.3, all components $\xi_{i}$ with $i>I$ satisfy the same inequality.

For $n$ large enough, we have that the difference between the first and the last component goes to zero exponentially fast in $n$. Therefore, the first component satisfies the inequality. Thus by Proposition B.3, all components satisfy the inequality.

Acknowledgements. One of the authors (FMT) thanks the Feigenbaum Lab at the Rockefeller University for its hospitality. His research (in 1988-1989) was carried out while he was on the faculty of Queens College, Flushing (NY). The research (in 1988-1989) of the other author (JJPV) was supported by DOE grant DE-AC-02-83-ER-13044 and carried out at Rockefeller University. We also acknowledge a discussion with Ben Bielefeld which led to a simplification of the proof of Theorem 3.1.

\section{References}

Angenent, S.B.: Monotone recurrence relations, their Birkhoff orbits, and topological entropy. Preprint, University of Wisconsin 1988

Aubry, S.: The twist map, the extended Frenkel-Kontorova model and the Devil's staircase. Phys. 7D, 240-258 (1983)

Bangert, V.: Mather sets for twist maps and the geodesics on tori. Preprint 1986

Goroff, D.: Hyperbolic sets for twist maps. Ergodic Theory Dyn. Syst. 5, 337-339 (1985)

Greene, J.M.: A method for determining a stochastic transition. J. Math. Phys. 20 (6), 1183-1200 (1979)

Katok, A.: Some remarks on Birkhoff and Mather twist map theorems. Ergodic Theory Dyn. Syst. 2, 185-194 (1982a)

Katok, A.: More about Birkhoff periodic orbits and Mather sets for twist maps. Preprint, University of Maryland 1982b

Kook, H., Meiss, J.D.: Periodic orbits for reversible symplectic mappings. Preprint, University of Texas at Austin 1988

Leage, I., MacKay, R.S.: Badly ordered periodic orbits of the standard map. Preprint, Warwick University 1986

MacKay, R.S., Meiss, J.D.: Linear stability of periodic orbits in Lagrangian systems. Phys. Lett. A 98, 92-94 (1983)

Li, W., Bak, M.: Fractal dimension of cantori. Phys. Rev. Lett. 57, 6, 655-658 (1986)

Mather, J.N.: A criterion for nonexistence of invariant circles. Publ. Math. IHES, 153-204 (1986)

Tangerman, F.M., Veerman, J.J.P.: Asymptotic geometry of hyperbolic well-ordered cantor sets. J. Stat. Phys. 59 (1-2), 299-321 (1990)

Veerman, J.J.P., Tangerman, F.M.: On Aubry Mather sets. Phys. D 46, 149-162 (1990)

Veerman, J.J.P., Tangerman, F.M.: Renormalization of Aubry Mather Cantor sets. J. Stat. Phys. 56 (1-2), 83-98 (1989)

Wilf, H.S.: Mathematics for the physical sciences. New York: Dover 1978

Communicated by T. Spencer 
